\title{
A MODEL TO SIMULATE THE RADIATIVE TRANSFER OF FLUORESCENCE IN A LEAF
}

\author{
Feng Zhao ${ }^{1, *}$, Qianqian $\mathrm{Ni}^{1}$ \\ ${ }^{1}$ School of Instrumentation Science and Opto-Electronics Engineering, Beihang University, Beijing 100191, PR China - \\ zhaofeng@buaa.edu.cn
}

KEY WORDS: Leaf optical properties, Chlorophyll fluorescence, Radiative transfer, Monte-Carlo method, Photon packet, Ray tracing

\begin{abstract}
:
Light is reflected, transmitted and absorbed by green leaves. Chlorophyll fluorescence (ChlF) is the signal emitted by chlorophyll molecules in the leaf after the absorption of light. ChlF can be used as a direct probe of the functional status of photosynthetic machinery because of its close relationship with photosynthesis. The scattering, absorbing, and emitting properties of leaves are spectrally dependent, which can be simulated by modeling leaf-level fluorescence. In this paper, we proposed a Monte-Carlo (MC) model to simulate the radiative transfer of photons in the leaf. Results show that typical leaf fluorescence spectra can be properly simulated, with two peaks centered at around $685 \mathrm{~nm}$ in the red and $740 \mathrm{~nm}$ in the far-red regions. By analysing the sensitivity of the input parameters, we found the MC model can well simulate their influence on the emitted fluorescence. Meanwhile we compared results simulated by MC model with those by the Fluspect model. Generally they agree well in the far-red region but deviate in the red region.
\end{abstract}

\section{INTRODUCTION}

Leaves are the most important organs of most vascular plants (Hirokazu, 2013). The interaction of electromagnetic radiation with plant leaves, e.g., reflection, transmission, absorption, and emission of chlorophyll fluorescence (ChlF), depends on the chemical and physical properties of the leaves. ChlF can be used as a direct probe of the functional status of photosynthetic machinery because of its close relationship with photosynthesis (Porcar-Castell et al., 2014).

Two analytic models, FluorMODleaf (Pedrós et al., 2010) and Fluspect (Vilfan et al., 2016), can calculate the radiative transfer (RT) of chlorophyll fluorescence in plant leaves.

In this paper, the radiative processes of fluorescence scattering and absorption in a leaf were simulated by adopting the MonteCarlo (MC) ray-tracing technique. The performance of the model is evaluated by comparisons with the Fluspect model.

\section{DESCRIPTION OF THE MODEL}

\subsection{Representation of the leaf}

In this newly proposed MC model, leaf is supposed as a semiinfinite slab, with a given thickness $d$ in vertical direction, and infinite extensions in horizontal direction. Leaf is considered to be composed as three parts: the upper and lower boundaries (epidermis layers), and the mesophyll layer. The interaction centers, which can absorb or scatter photon packet, are homogeneously distributed in the leaf mesophyll layer. However, there are no interaction centers in the upper and lower boundaries. So photons can be reflected or transmitted, but not absorbed in these epidermis layers. The Cartesian coordinate system is adopted with the upper left corner of the leaf as the origin (Fig. 1). In vertical dimension, the $\mathrm{z}$-axis is the normal of the leaf upper boundary pointing toward the inside of the leaf mesophyll. The upper boundary of the leaf is set equal to zero, and the lower boundary is set equal to $d$. Leaf's lengths in $\mathrm{x}$ and $y$ axes are $3 \mathrm{~cm}$. When photon packet leaves the leaf from one side, it is supposed to enter the leaf from the opposite side with the same direction to simulate infinite extensions in horizontal direction.

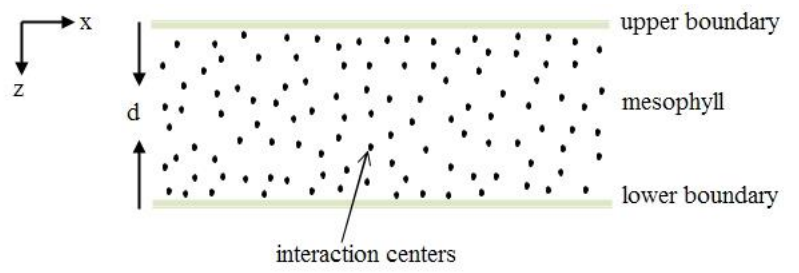

Figure 1. The modelled leaf with homogeneously distributed interaction centers within the thickness $d$

\subsection{Propagation of photon packet}

When a photon packet is incident perpendicularly on a given point in the leaf upper boundary, firstly part of the photon packet will be specularly reflected by the leaf surface. The rest of it will enter the leaf mesophyll and propagate further. The free path length (step size) $s$ of the photon packet before interaction is decided by a uniform random number between 0 and 1 and leaf optical properties. The step size $s$ is calculated by the Beer-Lambert law and an interaction coefficient as $\mu_{t}=\mu_{s}$ $+\mu_{a}$ where $\mu_{s}$ and $\mu_{a}$ are the scattering and absorption coefficients, respectively. The step size $s$ is then compared with the distance between the current photon location and the boundary of the mesophyll in the direction of the photon propagation, $s p$. If $s$ is greater than $s p$, we move the photon packet to the boundary without changing the direction of propagation, and compute the probability of the photon packet

\footnotetext{
* Corresponding author
} 
being internally reflected or transmitted. If the photon packet is internally reflected, it will propagate in the mesophyll in the direction of reflection. If it is transmitted, it will be scattered out of the lower leaf boundary and contribute to the leaf transmittance. Then a new photon packet is generated and traced again.

If $s$ is smaller than $s p$, the photon packet propagates in the mesophyll and moves a distance of $s$. Then it hits a reaction center, with part of its energy absorbed and the rest of its energy scattered in a new direction. The scattered direction of the photon packet is determined by the Henyey_Greenstein phase function. If the new step size of the scattered photon packet $s$ is still smaller than the new $s p$, it repeat the above process. If the scattered photon packet reaches the upper boundary, it will contribute to the leaf reflectance; or if it reaches the lower boundary, it contributes to the leaf transmittance. For either case, we terminate the tracing of it, and start tracing a new photon packet again.

Absorbed energy in every step can excite fluorescence and emit fluorescent photon packet, which experiences the similar tracing process above for non-fluorescent photon packet. The energy of the fluorescent photon packet equals to the absorbed energy multiplied by the fluorescent source function. We assume that fluorescent photon packet is excited isotropically: emission direction of the fluorescent photon packet follows the spherical distribution. For the fluorescent photon packet with a given emission direction, we can trace it just as non-fluorescent photon packet. We trace the fluorescent photon packet until: it escapes from the leaf upper boundary, which contributes to the leaf backward fluorescence; or it escapes from the leaf lower boundary, which contributes to the leaf forward fluorescence; or its energy is lower than a preset threshold. Then we return to tracing the non-fluorescent photon packet that emits the fluorescent photon packet. We repeats the whole process until the termination of the tracing of the non-fluorescent photon packet. A technique called Russian roulette is used to terminate a photon packet when its energy falls below a preset threshold.

There are two conditions to terminate the tracing of the nonfluorescent/fluorescent photon packet: 1) it is scattered out of the leaf upper or lower boundary; 2) it does not survive the Russian roulette. By tracing large amount of photon packets, e.g. 100000, the simulation can converge to a stable solution. Fig. 2 shows the flowchart of the MC model for the simulation of RT for a leaf, which has been implemented in C.

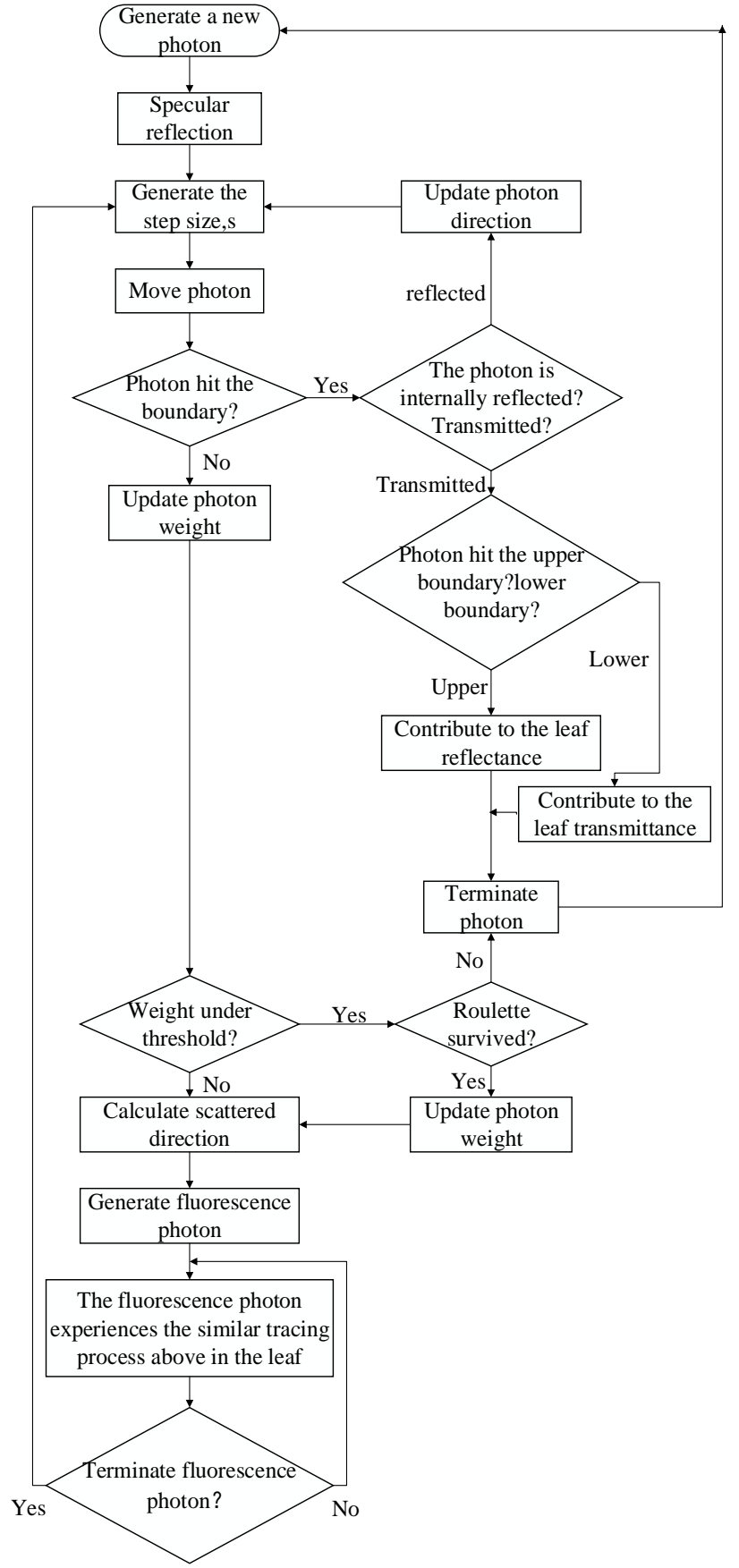

Figure 2. Flowchart of the MC model for the simulation of RT for a leaf

\section{RESULTS AND DISCUSSION}

\subsection{The input parameters of the MC model}

Table 1 shows the input parameters of the MC model and some of their standard values. Leaf thickness changes with plant types. The scattering anisotropy parameter, $g$, ranges from -1 (maximum backward scattering) to 1 (maximum forward scattering), with 0 representing isotropic scattering. Spectral scattering and absorption coefficients, $\mu_{s}$ and $\mu_{a}$, are spectrally dependent, and are determined by $d$ and $g$. In practice, $\mu_{s}$ and $\mu_{a}$ are fitted by minimizing the difference between simulated leaf reflectance/transmittance and measured ones with given $d$ and $g$. The ranges of excitation and emission wavelengths are from 400 to $750 \mathrm{~nm}$, and 640 to $850 \mathrm{~nm}$, respectively. Fluorescence 
quantum yield for photosystem I, $\Phi^{P S I}$, is relatively stable, while fluorescence quantum yield for photosystem II, $\Phi^{P S I I}$, is more variable and changes with photochemistry (Zhao et al., 2015).

\begin{tabular}{|c|c|c|c|}
\hline Parameter & Symbol & unit & $\begin{array}{c}\text { Standard } \\
\text { Value }\end{array}$ \\
\hline Leaf thickness & $d$ & $\mathrm{~cm}$ & 0.0235 \\
\hline $\begin{array}{c}\text { The scattering anisotropy } \\
\text { parameter }\end{array}$ & $g$ & - & 0.0 \\
\hline $\begin{array}{c}\text { Spectral scattering } \\
\text { coefficient }\end{array}$ & $\mu_{s}$ & $\mathrm{~cm}^{-1}$ & $\begin{array}{c}\text { spectrally } \\
\text { dependent }\end{array}$ \\
\hline $\begin{array}{c}\text { Spectral absorption } \\
\text { coefficient }\end{array}$ & $\mu_{a}$ & $\mathrm{~cm}^{-1}$ & $\begin{array}{c}\text { spectrally } \\
\text { dependent }\end{array}$ \\
\hline $\begin{array}{c}\text { Fluorescence quantum } \\
\text { yield for photosystem I }\end{array}$ & $\phi^{P S I}$ & - & 0.0023 \\
\hline $\begin{array}{c}\text { Fluorescence quantum } \\
\text { yield for photosystem II }\end{array}$ & $\phi^{P S I I}$ & - & 0.033 \\
\hline
\end{tabular}

Table 1. The input parameters for the MC model

\subsection{Simulation results of the MC model}

For a given intensity spectrum of the light source and values for a set of input parameters with standard values (Tab. 1), leaf directional-hemispherical reflectance and transmittance, and leaf backward and forward fluorescence, can be simulated by the MC model. Figure 3 shows the simulated leaf backward and forward fluorescence by the MC model. Results show that typical leaf fluorescence spectra can be properly simulated, with two peaks centered at around $685 \mathrm{~nm}$ in the red and $740 \mathrm{~nm}$ in the far-red regions.

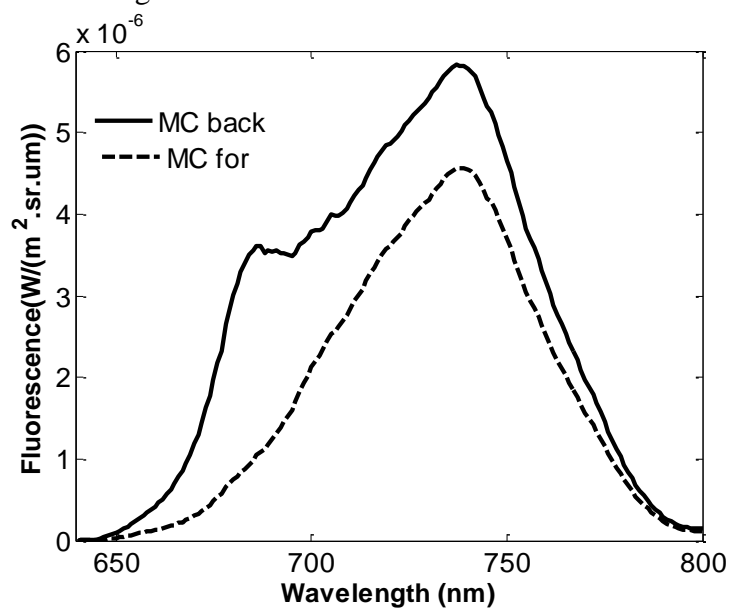

Figure 3. Simulated leaf backward (MC back) and forward (MC for) fluorescence by the MC model

\subsection{Sensitivity analysis of the input parameters}

Since a global sensitivity analysis for the MC model which is very time-consuming is not feasible, three parameters, $d, g$, and $\Phi^{P S I I}$, were chosen to vary separately with three typical values while other parameters were kept at their standard values.
3.3.1 Effect of the scattering anisotropy parameter on fluorescence emission: Three values of $g, 0,0.5,0.8$, which represent the scattering of the photon being from isotropic to more forward directions, were chosen to simulate fluorescence emission. With these values, the backward and forward fluorescence were simulated by the MC model, and shown in Fig. 4.

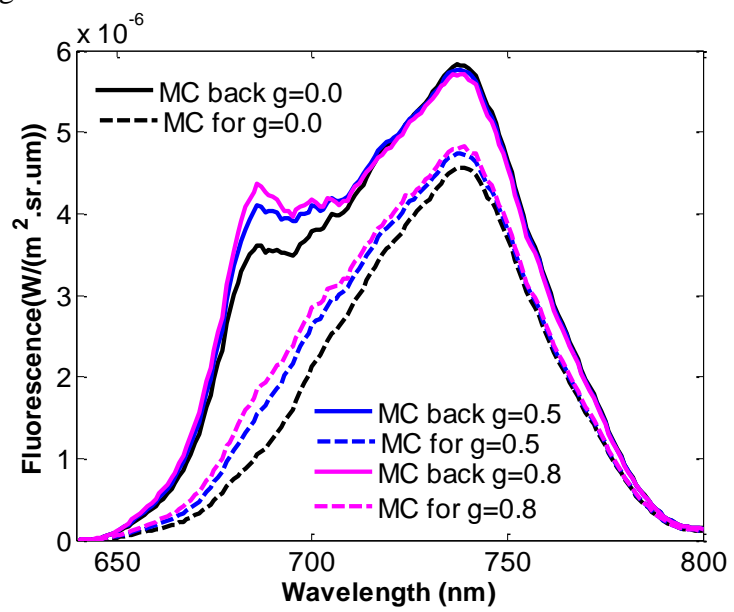

Figure 4. Simulated leaf backward (MC back) and forward (MC for) fluorescence by the MC model with $g$ of $0,0.5$, and 0.8

It can be seen that with the increase of $g$, backward and forward fluorescence also increases, especially for backward fluorescence around the red peak. Fluorescent photon is assumed to be emitted isotropically. The higher $g$ means the higher chance of the emitted fluorescent photon being scattered more in forward directions. Therefore, the path length of the fluorescent photon decreases after emission, which experiences less re-absorption during propagation. As a result, fluorescence escaping from the leaf upper and lower layers increases.

3.3.2 Effect of the leaf thickness on fluorescence emission: When leaf thickness increases, $d$, from $0.0135 \mathrm{~cm}, 0.0235 \mathrm{~cm}$, to $0.0325 \mathrm{~cm}$, no noticeable change is observed (Fig. 5). This is because its influence is transferred to $\mu_{s}$ and $\mu_{a}$, and it does not change the scattering direction of photon.

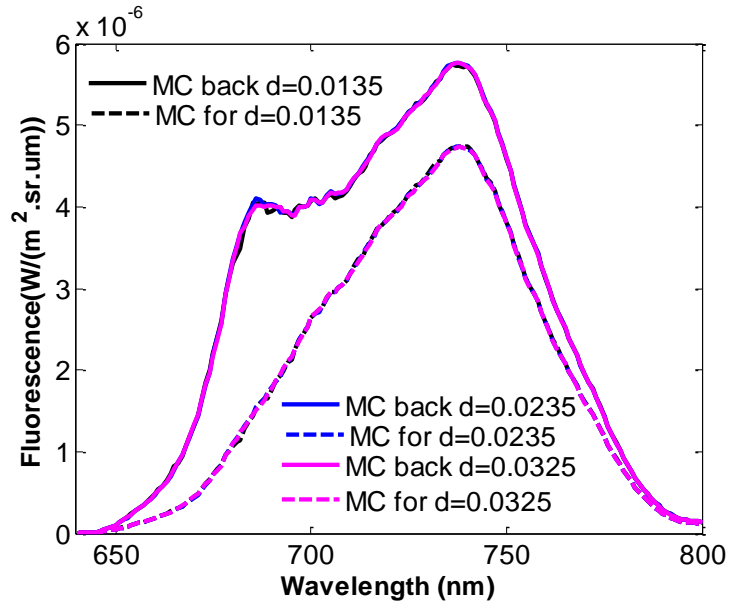

Figure 5. Simulated leaf backward (MC back) and forward (MC for) fluorescence by the MC model with $d$ of $0.0135,0.0235$, and 0.325 
3.3.3 Effect of fluorescence quantum yield of PSII on fluorescence emission: Figure 6 shows backward and forward ChlF for three values of $\Phi^{P S I I}, 0.033,0.067$, and 0.1 . It can be observed that both backward and forward ChlF increases with $\Phi^{P S I I}$, which is reasonable, since PSII contributes to both red and far-red peaks.

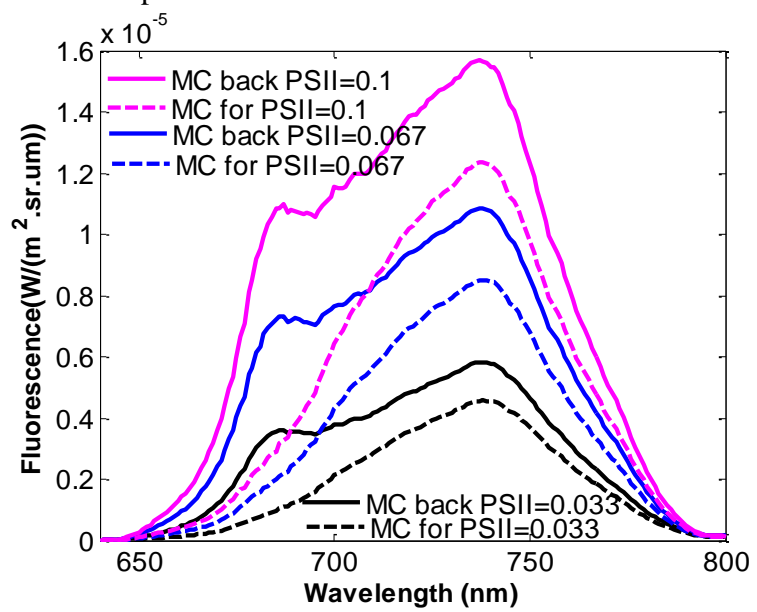

Figure 6. Simulated leaf backward (MC back) and forward (MC for) fluorescence by the MC model with $\Phi^{P S I I}$ of $0.033,0.067$, and 0.1

By analysing the sensitivity of the input parameters, we found the MC model can well simulate leaf fluorescence.

\subsection{Comparison with the Fluspect model}

The simulated results by the MC model were compared with those by the Fluspect model. To compare them under the same input conditions, the following procedure was adopted: the leaf reflectance and transmittance were simulated by the Fluspect model with its parameters (the first 5 parameters) shown in Table 2; $\mu_{s}$ and $\mu_{a}$ in the MC model were fitted by minimizing the difference between simulated leaf reflectance/transmittance by the two models with $g$ of 0.8 (which gives the best agreement of ChlF by the two models). Then the two models were compared with the determined parameters.

\begin{tabular}{|c|c|c|c|}
\hline Parameter & Symbol & unit & Value \\
\hline Leaf structure parameter & $\mathrm{N}$ & $\mathrm{cm}$ & 1.56 \\
\hline Chlorophyll a+b content & $C_{a b}$ & $\mu \mathrm{g} \cdot \mathrm{cm}^{-2}$ & 32.0 \\
\hline Total carotenoid content & $C_{c a}$ & $\mu \mathrm{g} \cdot \mathrm{cm}^{-2}$ & 9.42 \\
\hline Water content & $C_{w}$ & $\mathrm{~cm}^{-1}$ & 0.01 \\
\hline Dry matter content & $C_{d m}$ & $\mathrm{~g} \cdot \mathrm{cm}^{-2}$ & 0.005 \\
\hline $\begin{array}{c}\text { Fluorescence quantum } \\
\text { yield for photosystem I }\end{array}$ & $\phi^{P S I}$ & - & 0.0023 \\
\hline $\begin{array}{c}\text { Fluorescence quantum } \\
\text { yield for photosystem II }\end{array}$ & $\phi^{P S I I}$ & - & 0.033 \\
\hline
\end{tabular}

Table 2. The input parameters for Fluspect model

The simulated leaf reflectance and transmittance by these two models are compared in Figure 7. They agree closely with $\mathrm{R}^{2}$ and RMSE of 0.9995 and 0.0043 , and 0.9997 and 0.0037 , for reflectance and transmittance, respectively.

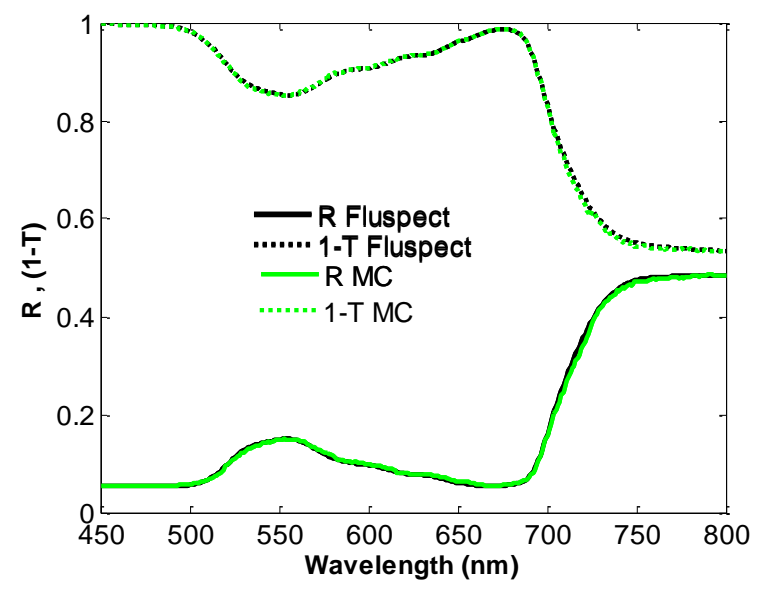

Figure 7 . The simulated leaf reflectance and transmittance by the MC model and the Fluspect model

Figure 8 shows the simulated backward and forward ChlF by these two models. The agreements between them around and after the far-red peaks are good. However, they deviate in the red regions, especially around the red-peak. This deviation may be caused by the weak re-absorption by the Fluspect model, which needs further investigation.

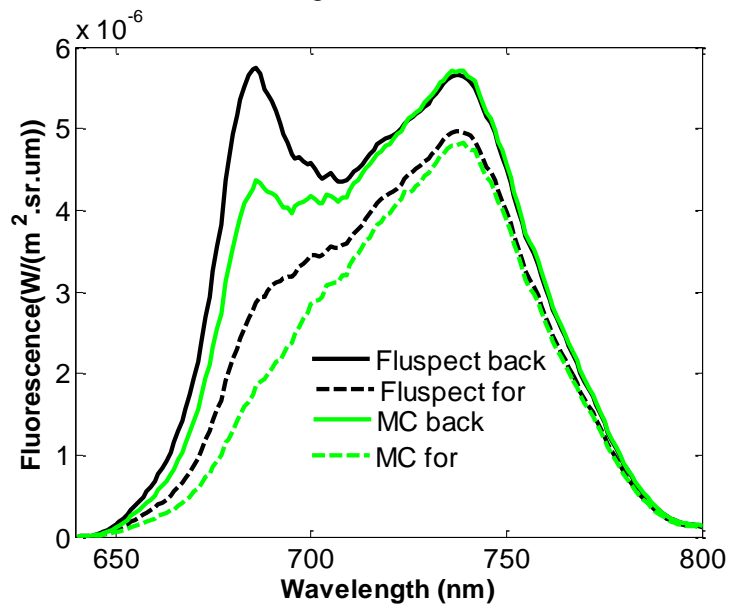

Figure 8 . The simulated leaf backward and forward ChlF by the MC model and the Fluspect model

\section{CONCLUSIONS}

In this paper, a MC model to simulate the RT of ChlF in the leaf was proposed. Sensitivity of the key input parameters of the model was analysed, which shows that the model can well reflect the influence of the parameters on ChlF. The comparison of ChlF simulated by the MC and Fluspect models revels the difference mainly comes from the red peaks. This difference may be caused by a relatively weak re-absorption simulated by the Fluspect model. More comparisons, especially with measured data, are needed to better evaluate the model, which is our future work.

\section{ACKNOWLEDGEMENTS}

This work was supported by the Chinese Natural Science Foundation (Projects 41771382). 


\section{REFERENCES}

Hirokazu, T., 2013. Leaf Development. The Arabidopsis Book. 11: e0163. doi:10.1199/tab.0163.

Pedrós, R., Goulas, Y., Jacquemoud, S., Louis, J., Moya, I., 2010. FluorMODleaf: a new leaf fluorescence emission model based on the PROSPECT model. Remote Sensing of Environment, 114, pp. 155-167.

Porcar-Castell, A., Tyystjärvi, E., Atherton, J., van der Tol, C., Flexas, J., Pfündel, E.E., Erhard, E., Moreno, J., Frankenberg, C., Berry, J.A., 2014. Linking chlorophyll a fluorescence to photosynthesis for remote sensing applications: mechanisms and challenges. Journal of Experimental Botany, 65(15), 40654095.

Vilfan, N., Tol, C. V. D., Muller, O., Rascher, U., Verhoef, W. , 2016. Fluspect-B: A model for leaf fluorescence, reflectance and transmittance spectra. Remote Sensing of Environment, 186, pp. 596-615.

Zhao, F., Guo, Y., Huang, Y., Verhoef, W., Tol, C. V. D., Dai, B., Zhao, H., Liu, G., 2015. Quantitative estimation of fluorescence parameters for crop leaves with bayesian inversion. Remote Sensing, 7(10), 14179-14199. 